\title{
LA POLÍTICA PÚBLICA PARA LA ATENCIÓN AL DESPLAZAMIENTO FORZADO EN EL DEPARTAMENTO DE CALDAS Y EN LOS MUNICIPIOS DE RIOSUCIO Y SAMANÁ (1997-2006)
}

\author{
MARIO HERNÁN LÓPEZ BECERRA ${ }^{1}$
}

Manizales, 2009-11-15 (Rev. 2010-05-20)

\section{RESUMEN}

A pesar de la magnitud del drama humanitario generado por el desplazamiento forzado en Colombia -y no obstante de la creación de un amplio marco jurídico para la atención a las personas en condición de desplazamiento-, la acción institucional se ha centrado en la atención humanitaria de emergencia; esta es una conclusión común en los balances nacionales acerca del cumplimiento de la política pública para la atención a la población en situación de desplazamiento.

Si bien se cuenta con buen número y alta calidad en las evaluaciones y balances nacionales de la política, es muy poco lo que se ha investigado acerca de la puesta en marcha de las políticas públicas en el Departamento de Caldas. A diferencia de las evaluaciones realizadas hasta ahora, la que se presenta en este trabajo no está orientada a cuantificar recursos destinados para la atención, busca más bien examinar la forma como se ha interpretado el problema por parte de los decisores públicos, la manera como se ha planificado la respuesta estatal y la forma como se ha propiciado o no la participación de la población afectada. A este tipo de aproximación se le puede definir como evaluación política de la política pública, para el período 1997-2006.

\section{PALABRAS CLAVE:}

Desplazamiento forzado, políticas públicas, conflicto armado, evaluación política, participación, población en situación de desplazamiento

\section{PUBLIC POLICY FOR THE ATTENTION OF VICTIMS OF FORCED DISPLACEMENT IN THE DEPARTMENT OF CALDAS IN THE MUNICIPALITIES OF RIOSUCIO AND SAMANA (1997-2006)}

\begin{abstract}
In spite of the magnitude of the humanitarian drama generated by the forced displacement in Colombia, and even though an inclusive legal framework has been created for the attention of displaced persons, the institutional actions have been focused on emergency humanitarian attention. This is a common conclusion in the national balances on the fulfillment of the public policy regarding the attention to displaced persons. Although the national evaluations and balances regarding the policy are numerous and of high quality, the research on the use of these public policies in the department of Caldas has been scarce. Differing from the evaluations carried out up to the present, the one presented in this article is not oriented towards the calculation of the resources destined for said attention; instead, it seeks to examine the manner in which the state response has been planned and the way in which the participation of the target population has been
\end{abstract}


promoted. This kind of approach can be defined as a political evaluation of the public policy for the 1997-2006 period.

\section{KEY WORDS}

Force displacement, public policies, armed conflict, political evaluation, participation, displaced persons.

\section{A MANERA DE INTRODUCCIÓN}

Desde su creación en el año 2004, la Red de Investigadores en Desplazamiento Forzado -REDIF- se propuso estimular la realización de trabajos de investigación con el propósito de avanzar en la construcción de enfoques, perspectivas, metodologías y procesos investigativos. Los encuentros nacionales de la Red han sido útiles para conocer e intercambiar trabajos investigativos sobre diversos tópicos del desplazamiento forzado, entre los cuales se cuentan algunos análisis de las políticas públicas. Una de las recomendaciones frecuentes ha sido la de realizar trabajos en las regiones que permitan examinar y valorar los alcances reales de las políticas.

Las evaluaciones nacionales de las políticas para la atención a las personas y comunidades sometidas al desplazamiento forzado, se han enfocado en la revisión del nivel de cumplimiento de las responsabilidades estatales en las distintas fases del desplazamiento; en efecto, luego de la expedición -en febrero del año 2004- de la sentencia T-025, las evaluaciones han buscado calificar la calidad y la cantidad de la respuesta gubernamental en el cumplimiento de las obligaciones claramente definidas por la Corte Constitucional. La dinámica que ha tenido el desplazamiento forzado interno y la fragilidad de la respuesta estatal, son constantemente examinadas y denunciadas provocando movilizaciones sociales alrededor de las demandas de los derechos de las víctimas.

En el caso de Caldas, la acción institucional para la atención a la población desplazada (durante el período abordado) se concentró en el nivel de la urgencia humanitaria. Como se sabe, una política centrada en la atención humanitaria de emergencia soslaya las causas de la problemática y se centra en la administración de la crisis.

Más allá de la contabilización de programas, proyectos y recursos y de los análisis de inversión, la evaluación de la política se puede centrar en otros asuntos relacionados con la forma como ésta se concibe, enfoca y expresa: ¿Cómo se interpretan las causas y dinámicas del desplazamiento forzado por parte de funcionarios y líderes sociales? ¿Cómo se enfocan las políticas públicas en Caldas (como departamento) y en los municipios de Samaná y Riosucio, en sus planes y programas de desarrollo? ¿Existe una dinámica propiciatoria para la participación en la toma de decisiones de quienes han sido sometidos al éxodo forzado?

El presente texto contiene un análisis aproximativo a las políticas públicas para la atención a las personas en condición de desplazamiento en la región caldense, con base en información obtenida de fuentes primarias y secundarias, para el período 1997-2006. Se trata de la presentación sucinta de los resultados de una investigación sobre el tema de las políticas públicas en materia de atención a las 
personas en condición de desplazamiento, realizada por el autor con el apoyo de la Vicerrectoría de Investigaciones y de Postgrados de la Universidad de Caldas.

\section{ACERCA DE LAS EVALUACIONES DE LA POLÍTICA PÚBLICA SOBRE DESPLAZAMIENTO FORZADO EN COLOMBIA}

Para el análisis de la política pública en materia de atención a las personas en situación de desplazamiento forzado en Colombia, se cuenta con buen número de documentos elaborados por organizaciones no gubernamentales y agencias internacionales, informes evaluativos de importantes organismos del Estado y memorias resultantes de múltiples seminarios y jornadas académicas sobre el tema. A través de ellos, se pueden establecer los alcances y resultados de la aplicación de las políticas públicas; en buena medida se trata de investigaciones y balances realizados por organizaciones no gubernamentales como la Consultoría para Derechos Humanos y el Desplazamiento (CODHES), la Conferencia Episcopal de Colombia, el Alto Comisionado de las Naciones Unidas para los Refugiados (ACNUR) y agencias del Estado como la Contraloría, las universidades y la Corte Constitucional. Una parte de estos trabajos ha presentado balances generales desde un enfoque de derechos (este enfoque busca determinar el grado de cumplimiento de las obligaciones estatales contraídas en el marco de las leyes y los acuerdos internacionales); otros trabajos apuntan a señalar los alcances y limitaciones del enfoque gubernamental de la política, así como las implicaciones que tiene para la población desplazada la expedición de la Ley 975 de 2005, conocida como Ley de Justicia y Paz.

En el año 2004, la Defensoría del Pueblo expuso algunas de las razones por las cuales se han presentado serias dificultades en la formulación y puesta en marcha de la política pública de atención al desplazamiento forzado en Colombia; en el documento de la Defensoría se relacionan asuntos fundamentales para comprender las limitaciones de la acción estatal derivadas de los problemas surgidos en la interpretación de la problemática y de la pobre disposición institucional para atenderla. En su momento, la Defensoría advirtió que la formulación y puesta en marcha de la política pública sobre desplazamiento forzado en Colombia, se nutrió básicamente de la experiencia de atención que paralelamente fue desarrollando el Estado y, por lo tanto -dice el mismo documento- es oportuno avanzar no solamente en la evaluación global del enfoque de la política pública en torno al fenómeno del desplazamiento, sino en la construcción de nuevas formas de entender y atender el fenómeno (Defensoría del Pueblo, 2004).

En una ponencia presentada en el encuentro nacional de investigadores en desplazamiento forzado convocado por REDIF -Pereira, 2006-, se examina la crisis en la que se encuentra la política pública; una crisis derivada de su extenso desarrollo normativo y su bajo nivel de ejecución real:

[...] tanto en la Sentencia T- 025 de 2004, como en el Auto 218 de 2006 de seguimiento de la misma Sentencia y en documentos de organismos consultivos como el CODHES, y en informes de organismos internacionales como el ACNUR, se ha puesto de relieve que la política pública construida y pensada para dar respuesta a la problemática del desplazamiento ha hecho crisis. Crisis que está relacionada con factores como la desarticulación del Sistema Nacional de Atención, la precariedad de los sistemas de información, la ausencia de 
monitoreo a los procesos de restablecimiento, la falta de indicadores de medición de impacto, entre otros. (Jaramillo, 2006)

En el mes de agosto del año 2002 se publicó el primer balance de la política de atención al desplazamiento interno forzado en Colombia 1999-2002 (Red de Solidaridad Social, ACNUR, 2002), este documento examina en cinco capítulos los distintos componentes de la política y plantea un conjunto de recomendaciones dirigidas a poner en funcionamiento el sistema nacional de atención a la población desplazada por la violencia (SNAIPD). La misma Agencia de las Naciones Unidas para los Refugiados realizó un segundo balance de la política pública que comprende el período agosto 2002 - agosto 2004; en las conclusiones se reconoce que se han producido avances concretos en los últimos años, particularmente en el reconocimiento por parte del Estado del desplazamiento interno forzado como el principal problema humanitario del país. Posteriormente, con el título de "Balance de la Política Pública para la Atención Integral al Desplazamiento Forzado en Colombia", la Agencia de la ONU para los Refugiados presentó el informe para el período 2004-2007 (ACNUR.2007), en el cual se valora positivamente el esfuerzo presupuestal de la nación, al mismo tiempo que se revelan como insuficientes los recursos asignados dada la magnitud del problema.

Tanto las evaluaciones del ACNUR, como los informes presentados por la Defensoría del Pueblo al Congreso de la República, los informes conjuntos de la Defensoría y la Contraloría y el trabajo elaborado por la Comisión Colombiana de Juristas y el Servicio Jesuita a Refugiados en Colombia (en el cual se realiza un importante seguimiento de la aplicación de las recomendaciones internacionales sobre desplazamiento forzado para el período 2004-2005), coinciden en señalar que la política pública presenta problemas graves en su aplicación en las diferentes fases del desplazamiento. Al respecto, la Comisión Colombiana de Juristas y el Servicio Jesuita a Refugiados en Colombia, también ha advertido que los programas gubernamentales de atención humanitaria y de restablecimiento socioeconómico resultan insuficientes frente a la enorme vulnerabilidad que sufren las personas en condición de desplazamiento. Estas mismas organizaciones señalan que el proceso de negociación con los paramilitares no ha contribuido a la prevención del desplazamiento ni a la protección de los derechos de la población en situación de desplazamiento:

Las víctimas están siendo doblemente victimizadas, los victimarios resultan protegidos por la impunidad y premiados económicamente, a través de subsidios de reinserción sin contrapartidas o de proyectos empresariales desarrollados en tierras usurpadas a la población desplazada [...] (p.8).

En conclusión, el panorama ofrecido por las evaluaciones de la política pública en materia de desplazamiento forzado, permiten identificar una situación de crisis en los terrenos de su formulación y aplicación, al mismo tiempo que impelen a la Sociedad y al Estado para acordar y poner en marcha un conjunto de políticas efectivas dirigidas a mitigar o resolver el conflicto, y garantizar el cumplimiento de los Derechos Humanos y las libertades individuales.

\section{PRECISIONES ACERCA DE LAS FUENTES DE INFORMACIÓN PARA EL ESTUDIO DE LAS POLITICAS PÚBLICAS EN CALDAS}

Diferentes estudios reconocen que es a partir del año 1997, en el marco de la profundización de la crisis cafetera, cuando se inician en el departamento de 
Caldas los desplazamientos forzados, tanto masivos como individuales. Con posterioridad a esta fecha se instalaron los comités de atención a la población desplazada y más adelante se iniciaron los trabajos de formulación de los planes integrales únicos. Por esta razón, para efectos del presente trabajo, se considera desde el año 1997 y hasta agosto de 2006, como rango temporal de la investigación.

Con respecto a las fuentes de información, vale la pena advertir que se accedió a un tipo información, a través de revisión documental y de entrevistas abiertas semiestructuradas, a funcionarios de diversas instituciones y a líderes de las organizaciones de personas en condición de desplazamiento, lo cual arrojó una información analizada en forma cualitativa a partir de categorías y subcategorías previamente diseñadas. Por consiguiente, la naturaleza del trabajo es estrictamente interpretativa. En el caso de la información sobre los planes de desarrollo, y otros documentos de interés, es necesario hacer algunas precisiones que ayudan a enmarcar las distintas consideraciones y conclusiones del trabajo.

Sobre los Planes de Desarrollo revisados:

\section{Samaná}

Aunque la investigación requería de la revisión de los Planes de Desarrollo municipales desde el año 1997 hasta el 2006, en el caso de Samaná no fue posible conseguir todas las versiones definitivas debido a inconvenientes que los funcionarios expresaron con relación a la administración anterior, la cual, por ser de un partido contradictor, no dejó información completa con respecto a su gestión. Se cuenta con el Plan de Desarrollo 2005-2007, una versión del Plan 2002-2004, que no especifica proyectos, y una propuesta de plan piloto para el Plan de Desarrollo 1999-2001.

\section{Riosucio}

De este municipio se pudo contar con los Planes de Desarrollo 1998-2000 y 2004-2007. Del período 2001-2003 no fue posible conseguir el documento por no encontrarse en la Secretaría de Planeación del municipio ni en el Archivo. Para este último período los análisis se basan en el informe de gestión del alcalde respectivo.

\section{Actas de Comités Departamental y Municipal:}

\section{Caldas}

Del comité departamental de atención a la población desplazada se revisaron actas de los años 2000, 2002, 2003, 2005 y 2006. Las del 2001 y 2004 no pudieron ser ubicadas ni en el Archivo ni en la oficina de la Unidad de Seguridad y Convivencia (la cual es la encargada de coordinar el comité por parte de la Secretaría de Gobierno). Vale decir, que la primera acta encontrada no es la primera del comité (el cual se conformó el 23 de diciembre de 1997 según Decreto 01434) y por no encontrarse numerada no es posible saber exactamente cuántas reuniones hubo antes de esa. No se encontraron actas de los años 1997,1998 y 1999. 


\section{Samaná}

No fue posible conseguir las actas de todas las reuniones que se han realizado por parte del comité de Samaná. La administración reconfiguró el comité expidiendo un Decreto el 5 de marzo de 2005.

\section{Riosucio}

Se pudieron obtener cuatro actas, correspondientes a las reuniones realizadas en el año 2004.

\section{CONCLUSIONES CENTRALES DE LA INVESTIGACIÓN}

\section{El juego del avestruz como interpretación del desplazamiento forzado en el Departamento de Caldas}

La ausencia de una interpretación fundamentada de las causas del desplazamiento forzado en los Planes de Desarrollo de Caldas, se relaciona con la carencia de marcos de interpretación y reconocimiento de los conflictos sociales, culturales y políticos en los instrumentos que se usan como base para la planificación. Es la preeminencia de una ideología del crecimiento económico, como sinónimo de desarrollo, que se traduce en la formulación de proyectos que promueven el crecimiento económico y la expansión de la infraestructura física como estrategias centrales para mejorar la base material y la eventual solución de los conflictos sociales.

En el caso del departamento de Caldas los Planes de Desarrollo y el enfoque en la gestión gubernamental se limitan, en el período examinado, a las acciones de atención humanitaria, reproduciendo lo que las evaluaciones nacionales han denunciado profusamente como un incumplimiento sistemático de los compromisos estatales en asuntos clave como el restablecimiento de la población desplazada.

No se encuentra una interpretación estructural de la dinámica del desplazamiento forzado en los diagnósticos en los cuales se apoyan los expertos en la formulación de los proyectos de atención a la población desplazada. Tampoco se reconoce que los conflictos generadores del desplazamiento forzado son, al mismo tiempo, causas y consecuencias de cambios en los usos del suelo, la presencia de cultivos de uso ilícito, la existencia de un territorio en disputa en el cual se localiza un proyecto de interés estratégico: el Proyecto Hidroeléctrico de la Miel-que representa el control hidroeléctrico de la región central de Colombiay la presencia significativa de municipios expulsores de población por razones asociadas a los conflictos entre actores armados como son Samaná, Pensilvania, Riosucio, Marquetalia, Marulanda, Manzanares, Neira, Salamina, así como el arribo de población desplazada a los municipios de Manizales, Samaná, La Dorada, Norcasia, Riosucio, Marquetalia, Supía.

Con relación a las interpretaciones sobre las causas de los conflictos y sus implicaciones sobre el desplazamiento forzado, el profesor Jaime Zuluaga (2005) ha señalado lo siguiente:

La cuestión del tipo de paz que queremos construir y el diseño de la sociedad posconflicto armado no son indiferentes de la comprensión de la naturaleza de 
conflicto que afrontamos. Un ejemplo claro es precisamente el problema del desplazamiento. Este no se entiende y no se puede afrontar adecuadamente sin tener clara la naturaleza del conflicto armado colombiano. (p.3)

Como se sabe por la experiencia de Colombia, las políticas exclusivamente dirigidas a la profundización de la guerra agravan la intensidad y magnitud de la problemática del desplazamiento forzado y las atenciones humanitarias de emergencia, aunque son claramente necesarias, constituyen el nivel más básico de atención a las crisis, movilizando hacia la pobreza extrema a las poblaciones afectadas.

\section{El municipio de Samaná: un caso de interpretación funcional del desplazamiento forzado}

Los documentos y entrevistas realizadas para la investigación, permiten señalar que en el caso del municipio Samaná, la gestión gubernamental para el período estudiado, se centró en la definición de procedimientos para la atención humanitaria de emergencia, lo cual orientó la gestión local hacia la consecución de recursos y la articulación institucional para la atención de la población.

Las características socio-económicas del municipio, su ubicación geográfica, la presencia de cultivos de uso ilícito, las condiciones de pobreza de la población (ubicado en el puesto 48 entre los 53 municipios del Eje Cafetero, en la medición del Índice de Desarrollo Humano, para el año 2004), y al mismo tiempo poseedor de importantes recursos naturales y territorio clave en proyectos hidroeléctricos, hacen que el municipio posea las características establecidas para los lugares expulsores de población: los desplazamientos se producen fundamentalmente en lugares geográficos donde coexisten fuertes estructuras de pobreza con abundancia de recursos estratégicos (Pérez, 2002).

Se puede afirmar que la interpretación generalizada acerca del desplazamiento forzado como un fenómeno asociado a la confrontación de actores armados en un territorio, no refleja por completo la naturaleza estructural del conflicto que le da origen. En consecuencia, hasta tanto no se identifiquen y exploren los diversos intereses existentes sobre el territorio, no se ponga sobre la mesa el problema de la distribución profundamente desigual de la tierra y, al mismo tiempo, se reconozca el asunto agrario como centro de los debates en Colombia, la política pública queda reducida a un conjunto de acciones dirigidas a administrar la crisis humanitaria sin avanzar hacia su superación definitiva.

\section{El municipio de Riosucio: un punto de vista estructural en la interpretación de las causas y dinámicas del desplazamiento forzado}

En este caso, la información examinada para el período 1997-2006 permitió identificar un conjunto de interpretaciones de las causas y dinámicas del desplazamiento forzado más cercanas a un análisis estructural, por parte de los responsables de la ejecución de la política para la atención a la población en situación de desplazamiento. En los documentos y entrevistas se encuentran explicaciones referidas a la posición geoestratégica del municipio, las condiciones de la tenencia de la tierra, las diferencias en la integración urbanorural y la pobreza generalizada de la población indígena. Es evidente que la gestión local en materia de atención a la problemática del desplazamiento buscó superar el voluntarismo y la caridad; se trata de un proceso más centrado en un enfoque de Derechos Humanos para lo cual se crearon instancias de coordinación interinstitucional con resultados importantes en materia de atención y prevención. 
La dinámica institucional del municipio de Riosucio permitió construir política pública local en materia de atención integral al desplazamiento forzado, esto se expresa en la existencia de escenarios de encuentro y discusión entre las organizaciones y personas desplazadas y los responsables de la ejecución de los planes y proyectos.

En este municipio los planes fueron diseñados desde un enfoque de derechos, apoyados en la capacidad de gestión de los funcionarios para articular esfuerzos y recursos internacionales, nacionales y departamentales.

Las interpretaciones que se hacen sobre el desplazamiento en los planes de desarrollo, y en los distintos documentos consultados, invitan a considerar que este municipio pudo, para la época, formular y poner en operación una política local para la atención al desplazamiento forzado adelantándose a las obligaciones que posteriormente le fueron asignadas a los entes locales, en el marco de la descentralización de las acciones de atención a la población desplazada.

\section{La quimera como horizonte en la participación de las víctimas}

Los obstáculos y dificultades para la participación de la población desplazada en las instancias de decisión de política pública, son dos de los aspectos más señalados en los balances nacionales realizados hasta ahora. En el seminario nacional de REDIF, realizado en noviembre de 2006, los representantes de las organizaciones de personas en condición de desplazamiento insistieron acerca de la necesidad de promover trabajos de investigación que aborden las dinámicas de las organizaciones de la población desplazada en Colombia. Los representantes de la organizaciones se refirieron a la necesidad de estimular investigaciones dirigidas a conocer las dinámicas de contestación a nivel local, regional y nacional, así como la realización de trabajos de acompañamiento y asesoría por parte de las universidades de manera que se pueda superar el coyunturalismo en el trabajo de las organizaciones y se logre avanzar hacia la generación de políticas. Algunos temas sugeridos en el seminario de REDIF para ser abordados fueron, entre otros, la reconstrucción sistemática de la memoria de los procesos de organización y las acciones de reclamación; lo étnico y el género en las organizaciones; los sentidos que construye la población que no está desplazada frente a la organización y la reivindicación de derechos.

En el mismo evento, la representación de la Mesa Nacional de Fortalecimiento de la Población Desplazada -MNFOPD- señaló los siguientes puntos como elementos críticos y teleológicos para las organizaciones:

- Las organizaciones deben transitar de ser promotores del asistencialismo a la demanda de derechos.

- Las organizaciones deben prepararse para realizar veedurías y seguimiento a la política pública.

- Algunas organizaciones son cooptadas por las clientelas políticas locales.

- Los sectores hegemónicos niegan espacios a las organizaciones que tienen propuestas alternativas.

- No hay una perspectiva de género y el papel de las organizaciones varía de acuerdo con la posición política de sus dirigentes.

- Las organizaciones nacen de manera inducida y tutelada como una estrategia para agilizar la gestión institucional (se trata de organizaciones que cumplen papeles logísticos en la circulación de suministros de la ayuda humanitaria). 
- Las organizaciones deben transitar por el camino de la autonomía tanto del Estado como de las Organizaciones no Gubernamentales.

- Se deben poner en marcha los procedimientos para la participación creados por la Sentencia T-025 y formar a quienes deben asumirlos.

En un trabajo realizado por estudiantes de la Universidad de Caldas, alrededor de cinco de las organizaciones de personas en condición de desplazamiento ubicadas en el departamento de Caldas y el nivel de cumplimiento de la política pública (Melchor \& Mejía, 2006), se concluye lo siguiente: "Algunos de los miembros de las organizaciones (asociaciones) de la ciudad de Manizales ADDEMAZ, CAMINANTES DE PAZ, ASODEPAZ y del municipio de Riosucio ASOVIVIR, no tienen apropiación del significado de la política pública; consideran que ésta no contribuye al restablecimiento de las personas en condición de desplazamiento". Esta distancia de las organizaciones con el concepto y contenido de la política, tiene su correlato en la acción social y en la capacidad de las organizaciones para demandar los derechos a la participación activa en los escenarios donde se toman las decisiones.

Caldas reproduce buena parte de los elementos planteados por los representantes de la Mesa Nacional de Fortalecimiento al advertir que el trabajo de las organizaciones es de carácter coyuntural, centrado en los aportes logísticos para la distribución de las ayudas; en suma, se trata de procesos organizativos signados por el caudillismo, sometidos a las presiones de las clientelas locales y caracterizados por la existencia de pocos espacios, escasos actores y ninguna estrategia.

\section{CONCLUSIÓN FINAL}

En Colombia, se podría reconocer la existencia de una política pública para atender el desplazamiento forzado si esta se definiera de manera convencional, es decir, si se entendiera sólo como un conjunto de acciones que realiza el Estado para resolver o mitigar una situación considerada como problemática. Si la definición tradicional se somete a juicio crítico, y en su lugar se plantea una definición más compleja de la política pública dirigida a la legitimación del Estado y la acción gubernamental -ampliando su capacidad para orientar la sociedad hacia el cumplimiento y disfrute pleno de los Derechos Humanos-, se modifica radicalmente la valoración que puede hacerse de la política en materia de desplazamiento forzado.

En teoría, el diseño y puesta en marcha de las políticas públicas está explicado por la necesidad de generar nuevas formas de relación entre la sociedad y el Estado, con ellas se busca mejorar el desempeño de las instituciones, generar capacidad para resolver problemáticas, de manera que al final se logre reforzar la confianza de la sociedad. Esta forma de definición de las políticas públicas permite contrastar la distancia entre lo propuesto y lo realizado, entre la interpretación que le da origen a una política y la dinámica social, económica, ambiental, cultural y política que hace o no coherente esa interpretación.

La política pública revela la dinámica conflictiva en que se construye la acción pública como producto de la interpretación de una problemática. Muestra la calidad del consenso construido, la capacidad para intervenir sobre las causas estructurales en una situación. En esta medida, una evaluación de la política supera la simple contabilidad de las acciones o la verificación del cumplimiento de metas e indicadores de eficiencia para convertirse en una manera de 
escudriñar en los intereses que están en juego a la hora de elaborar la agenda gubernativa.

Al examinar el tipo de interpretación que se le da a las causas y dinámicas del desplazamiento forzado, se encuentran diferencias significativas en los tres escenarios (Caldas, Riosucio y Samaná); en efecto, mientras en Caldas para el período 1997-2006 se soslaya la interpretación de las causas volcando de esta manera la acción institucional hacia la asistencia humanitaria, en el municipio de Riosucio se realiza una interpretación más compleja -en el sentido de las conexiones que se construyen entre los problemas sociales, económicos, y la dinámica del desplazamiento-, generando, de esta manera, una interpretación con aproximaciones estructurales al problema.

Podría concluirse que la política pública logra convertirse en un instrumento para la demanda social del cumplimiento de derechos -y es efectivamente útil para planificar la acción institucional-, cuando existe una interpretación construida sobre las causas y dinámicas del problema que requiere ser resuelto; de lo contrario, sólo se limita a ser una ruta para la administración de la crisis.

Al examinar la manera como ha sido incluida la población en condición de desplazamiento en los Planes de Desarrollo, los planes integrales únicos y los comités de atención de Caldas, Riosucio y Samaná, se constata la tendencia a considerarlos como población vulnerable; este tipo de política social desconoce la existencia de un conjunto de principios y derechos específicos de la población y las responsabilidades concretas que se derivan del reconocimiento legal de la situación del desplazado. A partir de la Sentencia T-025, la política pública está basada en un enfoque de derechos; en consecuencia, la acción de las autoridades debe dirigirse a reconocer a la persona en situación de desplazamiento como portadora de ellos y, por lo tanto, la gestión de las autoridades debe orientarse a garantizarlos de una manera coherente, diferencial y progresiva mediante acciones afirmativas; esto implica, como lo advierten algunos conferencistas de la Cátedra UNESCO -Bogotá, 2006-, plantear una respuesta desde el principio de responsabilidad y no tanto de solidaridad, como se ha realizado hasta ahora. La concentración de esfuerzos y recursos en Caldas, Riosucio y Samaná en el componente de emergencia de la atención humanitaria, los ubica en un momento anterior a la Sentencia y a los compromisos establecidos por la Ley de Justicia, Paz y Reparación.

Dados los acuerdos y negociaciones del gobierno con los grupos paraestatales, se ha puesto sobre la mesa la necesidad de impulsar nuevos enfoques para buscar la reparación de los daños causados a las víctimas, esto supone generar estrategias y recursos para la inversión social y la reparación en el marco de la Justicia Restaurativa; este tipo de justicia hace énfasis en la reparación de los daños. De avanzar el proceso de desmovilización en el país, se requerirá que los planes y otros procesos e instancias de planificación local incorporen programas de Justicia Restaurativa; no sobra decir que sobre estos aspectos centrales para la sociedad y la superación del conflicto, no se tienen previstas interpretaciones ni enfoques ni procesos de participación en las áreas consideradas en este estudio. 


\section{BIBLIOGRAFÍA GENERAL DE LA INVESTIGACIÓN}

- ACNUR. Balance de la política pública de prevención, protección y atención al desplazamiento interno forzado en Colombia. Agosto 2002 agosto 2004.Bogotá. 2004

- $\quad$ Balance de la política de atención al desplazamiento interno forzado en Colombia 1999-2002. Bogotá. 2002

- Balance de la Política Pública para la Atención Integral al desplazamiento Forzado en Colombia. Enero 2004 - abril 2007. Bogotá. 2007.

- ACNUR - CODHES. (2001). Desplazamiento forzado interno en Colombia: Conflicto, Paz y Desarrollo.

- Alarcón Flores, Luis Alberto. (2000). La cuestión social: reformar para excluir. V Congreso Internacional del CLAD sobre la Reforma del Estado y de la Administración Pública, Santo Domingo, Rep. Dominicana. En, http://unpan1.un.org/intradoc/groups/public/documents/CLAD/clad00 38413.pdf

- Arango, Carolina. (2006). Una revisión de las políticas públicas sobre desplazamiento forzado con énfasis en el restablecimiento. Universidad de Caldas. Documento inédito.

- Arboleda González, José Ubernel. (2003). Memorias del seminario internacional Análisis de Experiencias en Restablecimiento de la Población en Situación de Desplazamiento. Bogotá.

- Bello A., Martha Nubia. (2005). Restablecimiento. Entre retornos forzado y reinserción precaria. En El desplazamiento en Colombia. Regiones, Ciudades y Políticas Públicas. REDIF.

- Bello A., Martha Nubia y Villa M., Martha Inés (comps). (2005). El desplazamiento en Colombia. Regiones, Ciudades y Políticas Públicas. REDIF, ACNUR, Universidad Nacional, Corporación Región.

- Castrillón Sánchez, Pedro Pablo y Palacio, María Cristina. (2005). Conflicto Armado en el Eje Cafetero. En Desplazamiento en Colombia. Regiones, Ciudades y Políticas Públicas. REDIF.

- CODHES - Consultoría para los Derechos Humanos y el Desplazamiento. (2004)¡Como el cangrejo! El gobierno frente al desplazamiento. En Plataforma Colombiana de Derechos humanos, Democracia y Desarrollo. Reelección: El embrujo continúa. Segundo año de gobierno de Álvaro Uribe Vélez. Bogotá. 2004

- Comisión Colombiana de Juristas y el Servicio Jesuita a Refugiados Colombia. (2005). Seguimiento a la aplicación de las recomendaciones internacionales sobre desplazamiento forzado en Colombia 2004-2005. Bogotá.

- Corporación Jurídica Yira Castro. (2006, Nov.). En Caja de herramientas, Número 119. Bogotá. 2006.

- DANE, SMP, Gobernación de Caldas. (2005, Agosto). Análisis Demográfico y Diagnóstico Social de Caldas.

- Defensoría del Pueblo. (2004). Políticas Públicas y Desplazamiento: Una reflexión desde la experiencia. Bogotá.

- En Samaná piden que no los olviden. (2007, Feb. 26). La Patria.

- García Palacios, Omar. (2005) Las Políticas Públicas: Productos del Sistema Político. Nicaragua. En http://www.ciudadpolitica.com

- Hardt Michel y Negri Antonio. (2004). Multitud. Guerra y democracia en la era del imperio. DEBATE. Barcelona. 2004

- Jaramillo Marín, Jefferson. (2006). La política pública sobre atención a población desplazada en Colombia. Emergencia, constitución y crisis de un campo discursivo. Pontificia Universidad Javeriana, Bogotá. Ponencia presentada en seminario REDIF. Pereira. 
- Loaiza Giraldo, Ana María, et al. (2005). La coordinación interinstitucional y la convergencia Estado - Sociedad Civil como alternativas para el fortalecimiento de los comités municipales de atención integral a la población desplazada. En revista De Paso. Rostros y Caminos. Número 1. Manizales: Gobernación de Caldas - Universidad de Caldas.

- Medellín Torres, Pedro. (2004, Jul.). Sobre las políticas públicas y el régimen político. CEPAL.

http://www.eclac.org/publicaciones/xml/5/15555/sps93_Icl2170.pdf

- Melchor, Doracelly y Mejía Ángela, Viviana. (2006). Nivel de cumplimiento de la política pública en materia de restablecimiento y procesos organizativos de cinco asociaciones de personas en condición de desplazamiento del departamento de Caldas (Caminantes De Paz, Addemaz, Asodepaz y Asovivir). Tesis de grado en Trabajo Social, Universidad de Caldas.

- Mesa Interinstitucional en torno al Desplazamiento Forzado por el conflicto armado en Caldas. Revista De Paso. Rostros y Caminos. Número 1. Diciembre de 2004-Julio 2005. Manizales: Gobernación de Caldas Universidad de Caldas.

- Muller, Pierre. (1998). Génesis y fundamento del análisis de políticas públicas. INNOVAR, Revista de Ciencias Administrativas y Sociales, Universidad Nacional. Bogotá. (2006). Las políticas públicas. Bogotá: Universidad Externado de Colombia.

- OACNUDH (Oficina en Colombia del Alto Comisionado de las Naciones Unidas para los Derechos Humanos), ACNUR, CODHES. (2001). Compilación sobre desplazamiento forzado. Normas, doctrina y jurisprudencia nacional e internacional.

- Pérez Murcia, Luis Eduardo. (2002). Desplazamiento forzado en Colombia 1995-1999. Una aproximación empírica a las relaciones entre desplazamiento, conflicto armado y desarrollo. En El desplazamiento forzado en Colombia: Compromisos desde la Universidad. Bogotá. 2002.

- Rincón, Rafael. (2006). Desplazados y reparación. Caja de Herramientas, Número 116.

- Roth D. André-Noel. (2002). Políticas Públicas. Formulación Implementación Evaluación. Colombia. Ediciones Aurora - Unicauca.

- (2006). Discurso sin compromiso. La política pública de derechos humanos en Colombia. Bogotá: Ediciones Aurora.

- Solarte Pazos, Leonardo. (2002). La evaluación de programas sociales en el Estado liberal. Facultad de Ciencias de la Administración. Universidad del Valle. VII Congreso Internacional del CLAD sobre la Reforma del Estado y de la Administración Pública, Lisboa, Portugal, 8-11 Oct.

- Tabucchi, Antonio. (1999). La gastritis de Platón. Anagrama. Barcelona.

- Van Parijs, Phillippe. (2001). Más allá de la solidaridad. Los fundamentos Éticos del Estado Bienestar y su Superación. En Ciudadanía y Derechos Humanos Sociales. ENS.

- Vargas Velásquez, Alejo. El estado y las políticas públicas. Almudena Editores. Bogotá. 1999.

- Villegas Arenas, Guillermo, López Becerra, Mario Hernán. (2006). La Gestión Ambiental de las Pymes en Manizales, el interés ambiental y el consumo socialmente responsable del empresario. Manizales: Universidad de Caldas.

- Zimerman, Héctor. (2001). Aparición y desarrollo de las políticas públicas. Primer Congreso Argentino de Administración Pública, Sociedad, Gobierno y Administración Pública, Rosario, 30 de agosto a $1^{\circ}$ de septiembre. En, http://aulas.blogia.com/temas/politicas-publicas.php 
- Zuluaga Nieto, Jaime. (2005). La guerra interna y el desplazamiento forzado. En Cátedra virtual desplazamiento forzado en Colombia. Universidad Nacional. Bogotá.

- http://www.codhes.org/

- http://www.vivalaciudadania.org/herramientas/Herra103.pdf

- http://www.acnur.org/pais/docs/1706.pdf

1. Profesor Universidad de Caldas. Departamento de Economía y Administración. 